\title{
Gefahr durch Adipositas wächst
}

\author{
Da es immer mehr dicke Menschen mit entsprechen- \\ den Folgerisiken gibt, werden die Rufe nach neuen \\ wirksamen Methoden zur Gewichtsabnahme lauter. \\ Und es kommen auch seltsame Verfahren auf.
}

Eine weltweit steigende Prävalenz von Übergewicht (BMI $\geq 25 \mathrm{~kg}$ / $\left.\mathrm{m}^{2}\right) /$ Adipositas $\left(\geq 30 \mathrm{~kg} / \mathrm{m}^{2}\right)$ zieht auch mehr Typ-2-Diabetiker nach sich, woran Prof. Andreas Hamann, Hochtaunus-Kliniken Bad Homburg, erinnerte. Denn Typ-2-Diabetes ist die am engsten mit Adipositas assoziierte Komorbidität. Untersuchungen mit über 19 Mio. Teilnehmern zufolge ist von 1975 -2014 der altersstandardisierte mittlere BMI weltweit von 21,7 auf $24,2 \mathrm{~kg} / \mathrm{m}^{2}$ bei Männern und 22,1 auf 24,4 kg/m² bei Frauen gestiegen [1]. Während die die globale Prävalenz von Untergewicht sank, nahm die von Adipositas beim Mann von 3,2 auf 10,8 \% und bei Frauen von 6,4 auf 14,9\% zu. Weltweit haben 2,3\% der Männer bzw. $5 \%$ der Frauen einen BMI $\geq 35$ und 0,64 \% der Männer bzw. 1,6\% der Frauen einen $\geq 40 \mathrm{~kg} / \mathrm{m}^{2}$. Setzt sich dieser Trend fort, wird 2025 weltweit mit einer Adipositasprävalenz von $>18 \%$ bei Männern bzw. $21 \%$ bei Frauen zu rechnen sein. Eine Metaanalyse von 239 prospektiven Studien mit $>10$ Mio. Teilnehmern hat das niedrigste Mortalitätsrisiko in der BMI-Kategorie von $20-25 \mathrm{~kg} / \mathrm{m}^{2}$ (HR 1,00) gefunden, mit einem leichten Anstieg bei BMI unterhalb dessen (HR 1,13 für BMI 18,5-20). Mit dem BMI stieg auch die Mortalität, bis zu einer HR von 2,76 bei BMI von $40-60 \mathrm{~kg} / \mathrm{m}^{2}$. Für Europa beträgt die HR pro $5 \mathrm{~kg} / \mathrm{m}^{2}$-Anstieg des BMI 1,39. Dabei ist die Steigerung des Mortalitätsrisikos altersabhängig [2].

Die meisten sorgen sich ums eigene Gewicht und versuchen, ab- oder nicht weiter zuzunehmen. Dies bestätigt eine systematische Analyse der Daten von > 1 Mio. Teilnehmern, die in einem Review ausgewertet wurden [3]. Die Erfolge der konservativen Adipositastherapie sind aber überschaubar, so Hamann. Wie Hausärzte die Motivation der Patienten zu einer Gewichtsabnahme mit einer niederschwelligen 30-Sekunden-Intervention steigern können, zeigt eine Studie in englischen Hausarztpraxen [4]. Von $>8.400$ gescreenten Patienten waren 1.882 adipös.Ihnen wurde beim Arztbesuch entweder nur der Rat gegeben, ab- oder an einem Gewichtsreduktionsprogramm teilzunehmen (Interventionsgruppe). Das Programm umfasste 12 Sitzungen (1 Stunde) im wöchentlichen Abstand. Die Interventions-Patienten nahmen in 1 Jahr ca. 2,43 kg ab. Im Vergleich hatten die Kontroll-Patienten im Mittel 1,04 kg verloren. Der Unterschied war statistisch signifikant. Auch internetbasierte Strategien sind eine kosteneffektive Verhaltensintervention zum Abnehmen [5]. Dabei scheinen persönliche Follow-up-Termine zur Sicherung des initialen Erfolgs nicht nötig, so Hamann. Es reiche der Kontakt zwischen Praxispersonal und Patient via Telefon oder E-Mail in loser Folge.

\section{Bariatrische Chirurgie}

Versagen konservative Abnehmstrategien, kann nach den aktuellen Leitlinien bei Patienten mit Adipositas Grad III (BMI $\geq 40$ $\left.\mathrm{kg} / \mathrm{m}^{2}\right)$ oder II (BMI $\left.\geq 35 \mathrm{~kg} / \mathrm{m}^{2}\right)$ mit Komorbiditäten (z.B. Diabetes Typ 2) die Indikation für interventionelle Verfahren gestellt werden [6]. Die Effekte der Adipositaschirurgie (z.B. Magenbypass, Schlauchmagen, Magenband) auf metabolische Komorbiditäten, v.a. auf Typ2-Diabetes, sind in vielen Studien sehr beeindruckend, so Hamann. Das Magenband, das an Bedeutung verloren hat und nur noch bis zum BMI von $45 \mathrm{~kg} / \mathrm{m}^{2}$ eingesetzt werden sollte, gilt als die bariatrische Methode mit dem geringsten perioperativen Risiko. Die Adipositaschirurgie kann zu einer Verbesserung oder gar Remission der diabetischen Stoffwechsellage führen und auch das makrovaskuläre Risiko und Mortalitätsrisiko günstig beeinflussen.

\section{Alternative Therapien}

Alternativen zur bariatrischen Chirurgie sind etwa das ability ${ }^{\circledR}$-System, eine Kombination von implantierbarem Magenschrittmacher mit transgastralem Sensor für die Messung der Nahrungsaufnahme und Akzelerometer (Aktivitätsmessgerät). Der Schrittmacher hat in einer Studie mit 160 Adipösen (mittlerer BMI $43 \mathrm{~kg} / \mathrm{m}^{2}$ ) bei $61,3 \%$ zu einer Abnahme von mind. $25 \%$ ihres Gewichts geführt, dem Magenband war er nicht unterlegen. Komplikationen waren signifikant seltener und weniger schwer.

Als PEG-ähnliche, wenn auch recht seltsam anmutende Alternative stellte Hamann das AspireAssist-System ${ }^{\circledR}$ vor. Es besteht aus einer endoskopisch implantierten perkutanen Gastrostomie und einem auf der Bauchdecke platzierten Ventil. Nach der Mahlzeit kann der Patient das Ventil öffnen und einen Teil des Mageninhaltes abfließen lassen. Damit können etwa 30\% der Kalorien eingespart werden. In einer Studie mit 207 Teilnehmern (BMI $35-55 \mathrm{~kg} / \mathrm{m}^{2}$ ) hatten die Patienten damit nach $1 \mathrm{Jahr}$ im Mittel $31,5 \%$ ihres Übergewichts verloren vs. 9,8\% in der Kontrollgruppe (Lebensstilberatung) [7]. Wie Hamann schmunzelnd kommentierte, könne der mit dieser Methode versorgte Patient, anders als nach bariatrischem Eingriff, noch genussvoll eine reichhaltige Mahlzeit essen, bevor er sich 20 Minuten später auf der Toilette übers Ventil eines Teils entledigt. Dagmar Jäger-Becker

\footnotetext{
Literatur

1. NCD Risk Factor Collaboration (NCD-RisCC), Lancet 2016, 387:1377-96

2. The Global BMI Mortality Collaboration, Lancet 2016, 388:776-86

3. Santos I et al. Obesity Reviews 2017, 18:32-50

4. Little $P$ et al, Lancet Diabetes Endocrinol 2016, 4:821-8

5. Aveyard P, Lewis A, Tearne S et al. Lancet 2016, doi:10.1016/S01406736(16)31893-1. Epub ahead of print

6. S3-Leitlinie "Prävention und Therapie von Adipositas", 2014. www.awmf.org 7. Am J Gastroenterol. 2017 Mar;112(3):447-57.
}

Quelle: DiabetesUpdate 17./18. März 2017 in Mainz 